\title{
Solar ultraviolet radiation exposure of South African marathon runners during competition marathon runs and training sessions: a feasibility study
}

\author{
Victoria Nurse $^{1}$, Caradee Y Wright ${ }^{1,2^{*}}$, Martin Allen $^{3}$ and Richard L McKenzie ${ }^{4}$ \\ ${ }^{1}$ Department of Geography, Geoinformatics and Meteorology, University of Pretoria, South Africa. \\ ${ }^{2}$ Climate Studies, Modelling and Environmental Health Research Group, Council for Scientific and Industrial \\ Research, Pretoria, South Africa. \\ ${ }^{3}$ Department of Electrical and Computer Engineering, University of Canterbury, Christchurch, New Zealand. \\ ${ }^{4}$ National Institute of Water \& Atmospheric Research, NIWA Lauder, Central Otago, New Zealand
}

* Corresponding author email: Caradee Y. Wright, Council for Scientific and Industrial Research, South Africa, PO Box 395, Pretoria, 0001, Email: cwright@ csir.co.za, Tel: +27 128413092.

\begin{abstract}
Marathon runners spend considerable time outdoors training for and participating in marathons. Outdoor runners may experience high solar ultraviolet radiation (UVR) exposure. South Africa, where running is popular, experiences high ambient solar UVR levels that may be associated with adverse health effects. This feasibility study explores the use of personal dosimeters to determine solar UVR exposure patterns and possible related acute health risks of four marathon runners during marathons and training sessions in Cape Town and Pretoria. Runners running marathons that started early in the day, and that did not exceed 4 hours, yielded low total solar UVR exposure doses (mean 0.093 SED per exposure period run, median 0.088 SED, range $0.062-0.136$ SED; average of $16.54 \%$ of ambient solar UVR). Training sessions run during early morning and late afternoon presented similar results.
\end{abstract} Several challenges hindered analysis including accounting for anatomical position of personal 
dosimeter and natural shade. To assess health risks, hazard quotients (HQs) were calculated using a hypothetical runner's schedule. Cumulative, annual solar UVR exposure-calculated acute health risks were low $(\mathrm{HQ}=0.024)$ for training sessions and moderate $(\mathrm{HQ}=4.922)$ for marathon runs. While these data and calculations are based on 18 person-days, one can measure marathon runners' personal solar UVR exposure although several challenges must be overcome.

Keywords: solar ultraviolet radiation, exposure, marathons, running, South Africa.

\section{INTRODUCTION}

South Africa, located on the southern portion of the African continent, receives relatively high levels of solar ultraviolet radiation (UVR) due mainly to its latitude, relatively clear skies and topography (1). The country is characterised by an interior plateau with elevations in excess of $1400 \mathrm{~m}$ in some regions. Exposure to sufficient solar UVR is important for human beings as it stimulates production of vitamin D. Excess exposure to solar UVR exposure can lead to adverse human health effects and is associated with acute and chronic health effects, for example, sunburn and skin cancer, respectively (2). Due to South Africa's latitude, climate and the relatively high amounts of solar UVR that is received at the surface, it is important to understand the public health risks and exposure patterns of South Africans to better plan for skin cancer prevention campaigns and sun awareness programmes. Sports and recreation have been identified as an area where effective sun protection methods may be implemented to reduce adverse health risks from excess personal solar UVR exposure (3). Marathon runners may potentially be exposed frequently to large cumulative exposure doses of solar UVR depending on their running training schedules' timing and duration and their participation in marathons during the course of a year. Five 
Spanish runners received a mean solar UVR exposure of 7.62 \pm 4.28 SED units (SED, standard erythemal dose unit, $1 \mathrm{SED}=100 \mathrm{Jm}^{-2}$ ) for every 5 days of training and the average hourly outdoor reading was $0.59 \pm 0.61 \mathrm{SED}$ (4). Their training sessions were during the evening and spore-film dosimeters were attached with Velcro straps to the wrist. Other studies have found much higher solar UVR exposures, for example, among triathletes (5). Due to the nature of their solar UVR exposure patterns for training and competitions, marathon runners have been identified as a group of athletes at risk of non-melanoma and melanoma skin cancer $(6,7)$.

Marathon running is a popular sport in South Africa and marathons, ranging from 21 $\mathrm{km}$ (half marathons) to $42 \mathrm{~km}$ (full marathon) and even ultra-marathons (>80 km) are run in various cities and locations. The aim of this study was to test the feasibility of measuring, analysing and interpreting the solar UVR exposure of four marathon runners in South Africa during marathon runs and training sessions. Each runner's personal solar UVR radiation exposure was measured and then analysed with regard to ambient (surface) solar UVB radiation, temperature, cloud cover, solar zenith angle and location. While the results of the runners' exposure and possible acute health risks are reported, the focus is on the feasibility of carrying out such a study among marathon runners in South Africa with applicability elsewhere in the world too.

\section{MATERIALS AND METHODS}

Procedures. Two marathon runners were asked to run two half-marathons $(21 \mathrm{~km})$ on the 16 February 2014 in Cape Town (Cape Peninsula Marathon and Half-Marathon run from Cape Town to Simon's Town therefore in a general southerly direction) and on the 23 February 2014 in Pretoria (Deloitte Marathon and Half-Marathon, starts and ends at Hofmeyer Park and route changes direction), each wearing a UVR dosimeter badge 
(described below), strapped to the upper outer arm and on the outside of clothing (most runners wore sleeve-less vests). The participants differed for each marathon. Each runner wore a UVR dosimeter badge from the start of the marathon to its finish. One of the marathon runners was asked to wear the UVR dosimeter badge during a two-week period of training on the days that he trained, i.e. 29 March, 1 April, 5 April and 8 April 2014. For each marathon and the two-week training period, data were collected from two UVR dosimeter badges worn by the runners as well as from the South African Weather Service (SAWS) meteorological station nearest to the location of the marathon that was equipped with an instrument to record ambient solar UVR (described below). Temperature, wind speed, wind direction, cloud cover and cloud type data were also obtained from the SAWS meteorological station. The solar zenith angles and sunset and sunrise times were obtained from the South African Astronomical Observatory (SAAO) database. The ambient UVR data were collected from SAWS (for the nearest SAWS instrument closest to the marathon sites in Cape Town and Pretoria) and used in this study for comparison against the marathon runners' personal UVR exposure. Research ethics clearance was granted by the Council for Scientific and Industrial Research Ethics Committee (64/2013) and the University of Pretoria Ethics Committee (EC140721-066).

Personal UVR badges. The UVR dosimeter badges were developed to measure personal exposure to solar erythemal UVR (290-400 nm) and have been described in detail elsewhere $(8,9)$. The UVR dosimeter badges are manufactured by the University of Canterbury Department of Electrical and Computer Engineering, Canterbury, New Zealand. They have been shown to be suitable instruments for use in large personal solar UVR exposure studies $(10,11,12)$. The main component of the dosimeter badge is a miniature solid-state detector which measures erythemally-weighted UVR. The detector response is electronically converted into a digital count (on a scale from 1 to 1024) that is proportional to 
the incident erythemally-weighted UVR irradiance. The detector is encased in a weatherproof PTFE enclosure which also acts as a diffuser to ensure that the angular response of the instrument is close to the cosine response of human skin (13). The badge is powered by a small lithium coin cell battery (CR 1616, $3 \mathrm{~V}$ ) and has a diameter of 35mm, thickness of $13 \mathrm{~mm}$, and weighs approximately $20.7 \mathrm{~g}$. The badges were set to record data every 60 seconds in day/night mode (programmed to record from 06h00-21h00) and they have enough on-board memory and battery capacity to store numerous days of data.

The badge counts measured by the UVR dosimeter badges were converted to SED units. First, the badge counts were summed for every half an hour for the marathon and the two-week training period that took place in Pretoria and every hour for the marathon that took place in Cape Town. This was done to match the recording intervals of the ambient UVR instruments in both cities (see below). The summed badge counts were then converted to SED units using the calibration equations that were obtained for each badge (see calibration of UVR badges below). The runner's solar UVR exposure was then compared to the measured SAWS ambient UVR on a horizontal surface for the marathons and training period. The UVR badge was worn on the runner's upper arm, on top of clothing, facing outwards and was attached with a Velcro strap. Unfortunately, no record was kept of the orientation of the UVR badge on the runner's arm in relation to the rising sun.

Ambient solar UVR data. The SAWS has six stations in South Africa that monitor ambient solar UVR. These stations are: Pretoria (situated on the Highveld), De Aar, Durban, Port Elizabeth, Cape Town and Cape Point. The two stations used in this study were Pretoria $\left(25.7^{\circ} \mathrm{S}, 28.2^{\circ} \mathrm{E}\right.$, altitude $\left.\sim 1340 \mathrm{~m}\right)$ and Cape Town $\left(34.0^{\circ} \mathrm{S}, 18.6^{\circ} \mathrm{E}\right.$, altitude $\left.\sim 42 \mathrm{~m}\right)$. The instruments used by the SAWS to measure ambient solar UVR are UVR biometers. These are a broadband radiometer 501 model (manufactured by the Solar Light Co.) which consist of a Robertson-Berger pattern UVR detector that is able to detect solar UVB radiation from the 
sun in the wavelength range $280-340 \mathrm{~nm}$ thereby approximating the erythemal action spectrum (14), a digital recorder that records the readings, and finally a control unit that oversees the system and logs the data. The readings are logged hourly by the Cape Town biometer and every 30 minutes by the Pretoria biometer. The logged readings at both sites are reported in minimal erythemal dose values $\left(\mathrm{MED}, 1 \mathrm{MED}=210 \mathrm{Jm}^{-2}\right.$ ) and these were converted to SED values (where $1 \mathrm{SED}=100 \mathrm{Jm}^{-2}$ ) $(15)$. The biometers are calibrated according to manufacturer's instructions.

UVR dosimeter badge calibration. The UVR dosimeter badges were calibrated by comparing the output of the UVR badge against the erythemally-weighted UVR measured by the UVR biometer at the SAWS in Pretoria. Calibration took place daily from 24 April to 29 April 2014 and from $07 \mathrm{~h} 00$ to $21 \mathrm{~h} 00$ daily. The two badges were calibrated by placing them on a flat surface next to the UVR biometer on the SAWS roof for six days. The badges were set to record data every 60 seconds from $7 \mathrm{~h} 00$ to $21 \mathrm{~h} 00$ local time daily. The equations that were developed for each of the two badges were in the form of $2^{\text {nd }}$ order polynomials (Figure 1) and were used to convert the UVR dosimeter badge data for the marathon and training runs to SED units. It has been shown previously that calibration in one season or location may not always be applicable for dosimeter measurements at other seasons or locations (12). This is because there is not a perfect match between the dosimeter spectral response and the erythemal action spectrum, and because of this the conversion factor between badge counts and UVR dose has some dependence on SZA and ozone amount. The SZA dependence is implicitly dealt with by fitting a $2^{\text {nd }}$ order polynomial in the calibration. Since departures from linearity are small (see Figure 1), these mismatch errors must also be small. Differences in ozone affect the measurements in a similar way (and the amount of ozone in the light path at any time is approximately proportional to $\sec (\mathrm{SZA}))$. During the calibration period from 24 April to 29 April in 2014, the ozone amounts ranged from 245 to 270 Dobson Units (DU), 
whereas during the deployments, they ranged from $\sim 250$ to 265 DU (satellite ozone data extracted from http://www.esrl.noaa.gov/gmd/grad/neubrew/OmiDataTimeSeries.jsp).

Because these differences are rather small, the calibrations remain valid. If ozone amounts were significantly different, then radiative transfer models would have been needed to calculate the effect of these ozone differences. Correction factors for dosimeter badges are similar to those needed for other broad-band meters, including that used as the reference here (13).

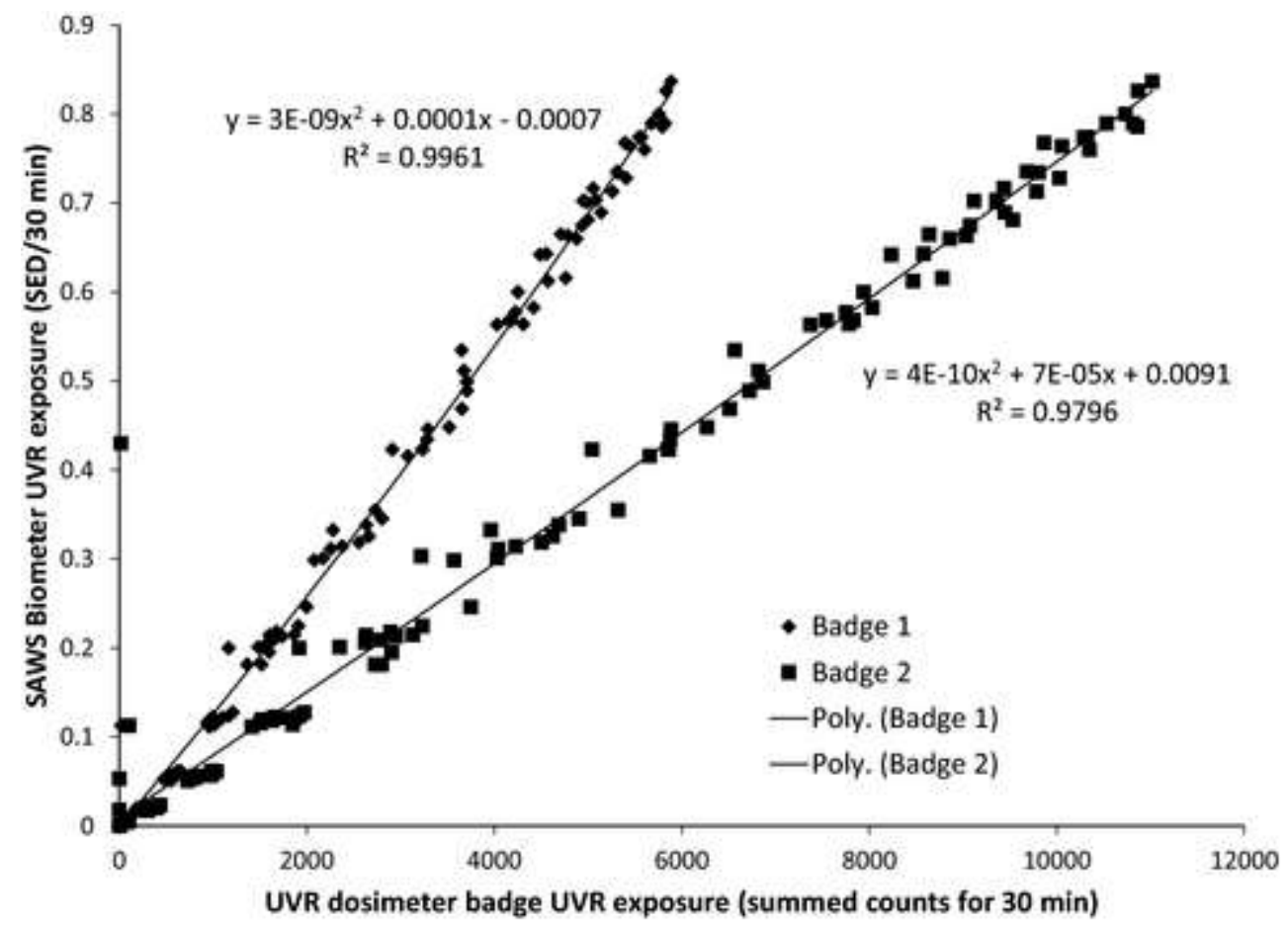

Figure 1. Calibration data for the two UVR dosimeter badges used in this study. The 30 minute measurements were undertaken at Pretoria over the period 24 to 29 April 2014. Dosimeters were set to record every 1 minute and these readings were summed for the 30-minute periods (therefore dosimeter counts exceed 1024) that the SAWS biometer measured solar UVR exposure. The equations are the $2^{\text {nd }}$ order polynomial fits for each dosimeter. The occasional zero values were likely due to shadows cast by humans, birds or insect passing nearby the dosimeters. 
Health risks assessed. Two approaches were used to assess acute health risks: the Fitzpatrick Skin Phototype (FST) Classification (16) and the Hazard Quotient (HQ) (17). The FST Classification (16) was used to analyse the acute associated health risks for solar UVR, particularly in terms of sunburn. Each marathon runner's skin type was determined and the total UVR exposure for the marathon was calculated. This information was then compared with the FST Classification defined amount of solar UVR exposure estimated to elicit a sunburn response on unprotected skin (16). The sunburn risk for each runner was then calculated.

Second, the HQ was calculated for non-carcinogenic, acute health effects (17). A typical year schedule for a marathon runner (Table 1, for full details of marathons run during a typical year see Supplementary Data, Table S1), including weekly training and marathons run, was drawn up for 2012 in Pretoria as a typical year (also the most recent year for which a full year's data were available at the time of the study). The training and marathon runs information was obtained from a marathon runner living in Pretoria. The marathon runner typically trained three days a week, either early in the morning or late in the afternoon for an average of 105 minutes.

Table 1. A South African marathon runner's typical schedule for 2012.

\begin{tabular}{|l|l|l|}
\hline Year 2012 & \multicolumn{1}{|c|}{ Marathons } & \multicolumn{1}{c|}{ Training } \\
\hline Number of days $(\mathrm{n})$ & \multicolumn{1}{|c|}{24} & \multicolumn{1}{c|}{152} \\
\hline Main running days & Saturdays/ public holidays & Alternating weekdays \\
\hline Some main events & $-\quad$ Two Oceans Marathon $(56 \mathrm{~km})$ & \\
& $-\quad$ Wally Hayward Marathon $(42 \mathrm{~km})$ & Not applicable \\
& $-\quad$ Nelspruit Race $(42 \mathrm{~km})$ & All year round \\
\hline Time of year favoured & $\begin{array}{l}\text { Mainly summer months except for the } \\
\text { Comrades Marathon run in May }\end{array}$ & $\begin{array}{l}\text { Early morning/late afternoon } \\
\text { (Training sessions span about } \\
\text { Time of day favoured }\end{array}$ \\
& $\begin{array}{l}\text { Early morning } \\
\text { (Marathons span 1-12 hours) }\end{array}$ \\
\hline
\end{tabular}

Typically, when long-distance marathons were run, for example with distances of 89 $\mathrm{km}, 72 \mathrm{~km}, 56 \mathrm{~km}$ and $50 \mathrm{~km}$, training would not take place a few days before and after the event. Marathons were run mainly on Saturdays, and times and distances varied (see 
Supplementary Data, Table S1). Ambient solar UVR data for Pretoria were obtained from the SAWS for 2012 in order for the HQ to be calculated. The HQ was calculated using the following formula, which has been adapted from the National Research Council (17):

$$
\begin{aligned}
& \text { Hazard quotient }(\mathrm{HQ})=\frac{\text { Average daily dose (SED units) }}{\text { Reference exposure level (SED units) }} \\
& \text { Where: } \quad \text { Average daily dose }=\frac{\text { UVR exposure (SED units) }}{\text { Number of days (n) }}
\end{aligned}
$$

The Reference Exposure Level (REL) was defined as 1.08 SED units (18). Before the HQ could be calculated, the training and marathon solar UVR exposure averages of all the runners in the study were calculated so that they could be applied in the HQ calculation. The marathon average HQ was calculated by taking the UVR dose obtained from the UVR dosimeter badges (after calibration) and dividing it by the corresponding ambient UVR dose obtained from SAWS for the same period (i.e., half-hour periods for Pretoria and one-hour periods for Cape Town) and multiplying it by 100 to obtain a percentage. This was done for both half-marathons that were monitored (total of four runners). The four percentages were then averaged to obtain the 'marathon solar UVR exposure' average amount. The training solar UVR exposure average was calculated using the same method.

The percentages for the marathon runs were then summed and divided by the number of days marathons took place to obtain the average daily marathon solar UVR exposure (in SED units/day). Thus, the calculated UVR exposure was divided by the number of days that marathons were run, in this case $n=24$ days. The calculated average daily dose was divided by the REL. This process was repeated for the training schedule for the year 2012 using the UVR exposure (obtained from the UVR dosimeter badge) for the two-week training period. The number of training days in a typical year was defined as $n=152$ days. 
For interpretation of the HQs, the following guidelines were applied from Lemly (19): A HQ $<0.1=$ no hazard risk; HQ between 0.1 and $1.0=$ low hazard risk; HQ between 1.1 and $10=$ moderate hazard risk; and HQ > $10=$ high hazard risk. These guidelines were used to assess the HQ results obtained for the marathon runs and training runs for a typical marathon runner during one typical year.

\section{RESULTS}

Marathon runner's solar UVR exposures: marathon runs. Results for all marathon runner's solar UVR exposure, for both competitions and training sessions, are provided in Table 2. Figures $2 \mathrm{a}$ and $2 \mathrm{~b}$ shows the marathon runners' solar UVR exposure plotted against the ambient solar UVR exposure for the same time periods (i.e. every 30 minutes) on 23 February in Pretoria. The ambient solar UVR peaked at 2.4 SED units per 30 minutes between $13 \mathrm{~h} 00$ and $13 \mathrm{~h} 30$. The first marathon runner's solar UVR exposure peaked at 0.012 SED units per 30 minutes between $8 \mathrm{~h} 00$ and $8 \mathrm{~h} 30$ (Figure 2a). This peak exposure occurred an hour later than that of the second marathon runner (peak at 0.025 SED units per 30 minutes between $7 \mathrm{~h} 00$ and 7h30) (Figure $2 \mathrm{~b}$ ).

Figure $2 \mathrm{c}$ depicts the first marathon runner's solar UVR exposure plotted against the ambient diurnal UVR for the same time periods (i.e. every 60 minutes) on 16 February in Cape Town. The ambient solar UVR peaked at 4.5 SED units per hour between $13 \mathrm{~h} 00$ and 14h00. The marathon runner received low solar UVR exposure while running, peaking at 0.022 SED units per hour between $10 \mathrm{~h} 00$ and $11 \mathrm{~h} 00$. The second marathon runner who took part in the half-marathon on 16 February in Cape Town had similar results (Figure 2d). The 

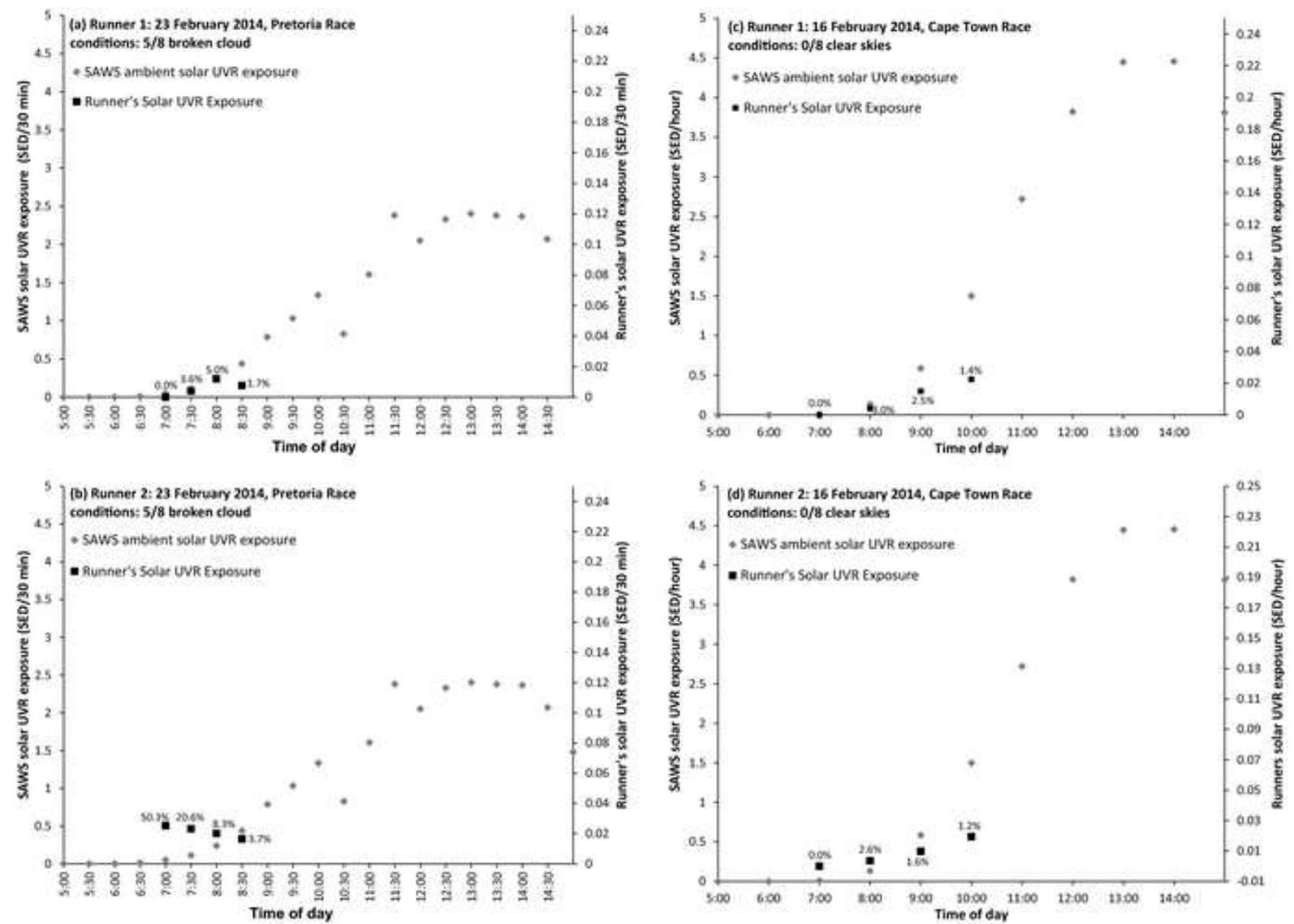

Figure 2. Solar UVR exposure measured by two competitors at each of the competition marathon runs compared with ambient UVR measured by the SAWS. Time periods of ambient solar UVR exposure measurements (SAWS data) differed between Cape Town and Pretoria, with 1 hour integrations for Cape Town, compared with 30 minutes integrations for Pretoria, however, this was accounted for in the data processing. The scale for the ambient solar UVR measurements (SAWS data) is on the y-axis-1(left hand side) and the scale for the runner's solar UVR exposure data is on the y-axis-2 (right hand side) (Please note that the runners scale is 20 times smaller than the SAWS ambient solar UVR exposure scale.)

marathon runner's solar UVR exposure peaked at 0.019 SED units per hour between $10 \mathrm{~h} 00$ and $11 \mathrm{~h} 00$.

We note that the total UVR dose available throughout the day was much greater than that of the runner exposure dose during the period of the marathons. For example, the total ambient solar UVR doses in Cape Town on the two days in question were 26.84 (23 
February) and 26.44 (16 February) SED, with peak values of $~ 8$ SEDs for the 2-hour midday period.

Meteorological conditions during marathon runs. Meteorological variables (Tables 3

and 4) were compared to try to account for any influences they might have had on the amount of solar UVR that the marathon runners received during the marathon running and the training sessions. On 16 February, the first half-marathon took place in Cape Town. No clouds were reported for that day. The temperature increased during the half-marathon, reaching $30.4^{\circ} \mathrm{C}$ by $10 \mathrm{~h} 00$. The wind speed also increased during the half-marathon, however, remained on average in a south-westerly direction. The half- marathon run on 23 February in Pretoria had different conditions. The temperature did increase during the morning and reached $25.4^{\circ} \mathrm{C}$ by $20 \mathrm{~h} 00$. However, the temperatures remained cooler when compared to the Cape Town half-marathon temperature. The wind speed remained fairly constant throughout the half-marathon, blowing in a general south-easterly direction. The half-marathon in Cape Town was run in conditions with no cloud cover, compared with the marathon in Pretoria, which had 5 octas (5/8ths) of cloud cover reported.

The sunrise and sunset times and the solar zenith angle play important roles in the amount of solar UVR reaching the Earth's surface. When comparing the two half-marathons on 16 February in Cape Town and 23 February in Pretoria, the sunrise time was much earlier in Pretoria than Cape Town. This led to a slightly longer exposure time of the marathon runners in Pretoria. However, the sunset times were much later in Cape Town. 
Table 3. Temperature, wind speed, wind direction and cloud cover of half-marathons monitored in Cape Town on 16 February 2014 and Pretoria on 23 February 2014 and two-week training session from 27 March to 08

April 2014.

\begin{tabular}{|c|c|c|c|c|c|c|c|}
\hline Date & Location & Hour & $\begin{array}{c}\text { Temperature } \\
\left({ }^{\circ} \mathrm{C}\right)\end{array}$ & $\begin{array}{c}\text { Wind } \\
\text { Speed } \\
(\mathrm{m} / \mathrm{s})\end{array}$ & $\begin{array}{c}\text { Wind } \\
\text { Direction } \\
\left(^{\circ}\right)\end{array}$ & $\begin{array}{c}\text { Wind } \\
\text { Direction }\end{array}$ & $\begin{array}{c}\text { Cloud } \\
\text { Amount } \\
\text { (octas) }\end{array}$ \\
\hline \multirow[t]{5}{*}{$\begin{array}{l}16 \\
\text { February }\end{array}$} & Cape Town & 06:00 & 23.8 & 2.9 & 224 & SW & \multirow[t]{5}{*}{ No Cloud } \\
\hline & & 07:00 & 23.2 & 2.8 & 249 & SWS & \\
\hline & & 08:00 & 25.3 & 2.8 & 237 & SWS & \\
\hline & & 09:00 & 28.5 & 3.1 & 228 & SW & \\
\hline & & $10: 00$ & 30.7 & 3.9 & 165 & SSE & \\
\hline \multirow{6}{*}{$\begin{array}{l}23 \\
\text { February }\end{array}$} & Pretoria & 06:00 & 19.5 & 1.3 & 72 & ENE & \multirow{6}{*}{$\begin{array}{c}5 / 8 \\
\text { 'Broken' }\end{array}$} \\
\hline & & 07:00 & 19.7 & 1.7 & 63 & ENE & \\
\hline & & 08:00 & 20.9 & 1.5 & 31 & NNE & \\
\hline & & 09:00 & 23.5 & 1.4 & 39 & $\mathrm{NE}$ & \\
\hline & & $10: 00$ & 25.4 & 1.6 & 26 & NNE & \\
\hline & & $11: 00$ & 26.4 & 1.5 & 35 & $\mathrm{NE}$ & \\
\hline \multirow[t]{3}{*}{29 March } & Pretoria & 06:00 & 14.0 & 1.2 & 40 & $\mathrm{NE}$ & \multirow{3}{*}{$\begin{array}{c}5 / 8 \\
\text { 'Broken' }\end{array}$} \\
\hline & & 07:00 & 14.2 & 0 & 0 & $\mathrm{~N}$ & \\
\hline & & 08:00 & 15.6 & 1.3 & 30 & NNE & \\
\hline \multirow[t]{3}{*}{1 April } & Pretoria & 06:00 & 13.5 & 1.1 & 40 & $\mathrm{NE}$ & \multirow{3}{*}{$\begin{array}{c}2 / 8 \\
\text { 'Few' }\end{array}$} \\
\hline & & 07:00 & 13.7 & 0 & 0 & $\mathrm{~N}$ & \\
\hline & & 08:00 & 15.3 & 0 & 0 & $\mathrm{~N}$ & \\
\hline \multirow[t]{3}{*}{5 April } & Pretoria & 06:00 & 13.3 & 1.9 & 90 & $\mathrm{E}$ & \multirow{3}{*}{ No Cloud } \\
\hline & & 07:00 & 13.0 & 1.8 & 120 & ESE & \\
\hline & & 08:00 & 14.0 & 1.3 & 140 & $\mathrm{SE}$ & \\
\hline \multirow[t]{3}{*}{8 April } & & 06:00 & 9.6 & 1.8 & 180 & $\mathrm{~S}$ & \multirow{3}{*}{$\begin{array}{c}2 / 8 \\
\text { 'Few' }\end{array}$} \\
\hline & & 07:00 & 9.3 & 1.7 & 160 & SSE & \\
\hline & & 08:00 & 11.2 & 1.6 & 100 & $\mathrm{E}$ & \\
\hline
\end{tabular}

Table 4. Sunrise times, sunset times and azimuth angle for the two half-marathons monitored on 16 February

2014 and 23 February 2014 and the two-week training session monitored from 29 March to 08 April 2014. The

Cape Peninsula Marathon and Half-Marathon run from Cape Town to Simon's Town was in a general southerly direction but the Pretoria Deloitte Marathon and Half-Marathon route direction changed regularly. It was not possible to determine the orientation of the UVR badge relative to the rising sun.

\begin{tabular}{|l|l|c|c|c|}
\hline Date & Location & Sunrise & Sunset & Azimuth Angle $\left(^{\circ}\right.$ ) \\
\hline 16 February 2014 & Cape Town & $06: 22$ & $19: 38$ & 106 \\
\hline 23 February 2014 & Pretoria & $05: 57$ & $18: 43$ & 101 \\
\hline 29 March 2014 & Pretoria & $06: 15$ & $18: 09$ & 86 \\
\hline 1 April 2014 & Pretoria & $06: 16$ & $18: 06$ & 84 \\
\hline 5 April 2014 & Pretoria & $06: 18$ & $18: 01$ & 82 \\
\hline 8 April 2014 & Pretoria & $06: 19$ & $17: 58$ & \\
\hline
\end{tabular}


Marathon runner's solar UVR exposures: training sessions. A series of training sessions was carried out from 27 March to 08 April 2014 in Pretoria. Four training runs took place during this period and all runs occurred early in the morning. The start times varied from 6 h00 to 8 h00. Figure 3a-d depicts the UVR dose training runs that took place in March and April. Low values of solar UVR were recorded for the training run on the 29 March (Figure 3a) and the highest SED value per 30 minute period that the runner received was 0.012 SED/30 minutes on this day. The total cumulative UVR exposure for this training session was 1.85 SED/training session on the 29 March. On the 1 April, low values of ambient solar UVR were also recorded (Figure 3b). The highest SED value was 0.002 SED / 30 minutes and the total cumulative UVR exposure received by the runner for this training session was 1.49 SED/training session. On both days, the maximum SED/training sessions received by the runners was similar; however, the total daily ambient solar UVR recorded for the days was slightly less on 01 April when there were less clouds when compared to 29 March.

The total UVR dose available throughout the day was much greater than that of the dose during the periods of time spent training. For example, the total ambient exposure doses on the two days in question (29 March and 1 April) were 17.94 and 17.86 SED respectively, with peak values of around 7 SEDs for the 2-hour midday period. This illustrates the importance of the early-morning starts in curtailing solar UVR exposure. 

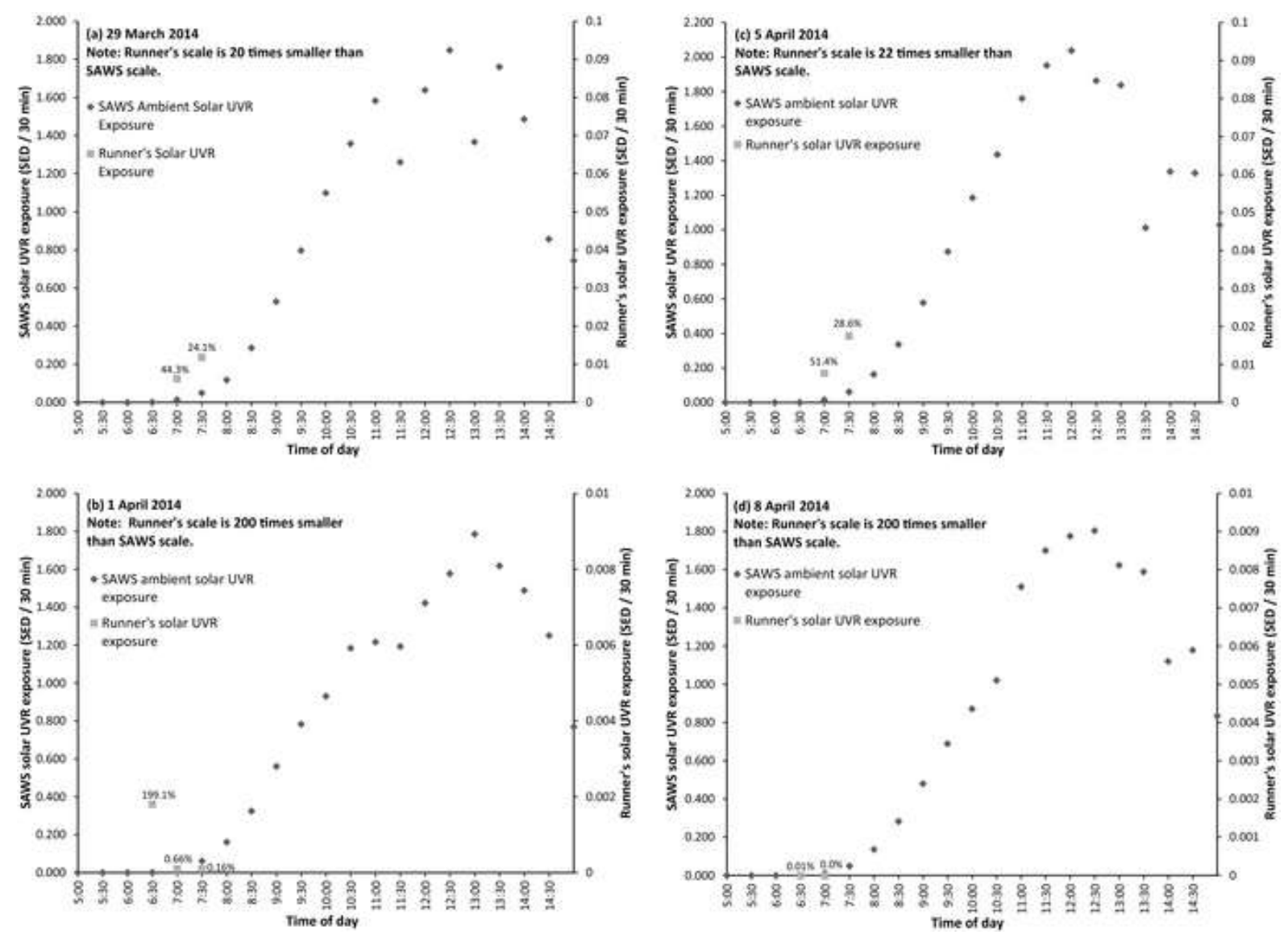

Figure 3. Marathon runner's solar UVR exposure compared to ambient solar UVR exposure (SAWS data) for training runs on the 29 March, 1 April, 5 April and 8 April 2014. The scale for the ambient solar UVR measurements (SAWS data) is on the y-axis-1(left hand side) and the scale for the runner's solar UVR exposure data is on the y-axis-2 (right hand side). (Please note that the runners scale differs compared to the SAWS scale and these differences are detailed on the figures.)

Meteorological conditions during training sessions. Atmospheric variables were compared to try to account for any influences they might have had on the amount of solar UVR that the marathon runner received during the training sessions (Tables 3 and 4). The training sessions that took place from 27 March to 08 April had varying conditions. On 29 March, the temperature increased from $14.0^{\circ} \mathrm{C}$ to $15.6^{\circ} \mathrm{C}$ by $8 \mathrm{~h} 00$. This is a lot cooler when compared with the temperatures measured during the half-marathons run in February 2014. The cloud cover also played a role on this day, with 5 octas being measured. The wind speed remained fairly constant in a general north-easterly direction. On 1 April, the temperature 
reached $13.7^{\circ} \mathrm{C}$ by $7 \mathrm{~h} 00$, which was similar to 29 March. There was also very little wind that morning and only 2 octas of cloud was reported for that day. On 5 April, the temperature reached $13.0^{\circ} \mathrm{C}$ by $7 \mathrm{~h} 00$ and no cloud was reported. The wind speed decreased from $6 \mathrm{~h} 00$ to 8h00 and remained in a general easterly direction. On 8 April, the temperature was slightly cooler at $7 \mathrm{~h} 00$ at only $9.3^{\circ} \mathrm{C}$. Only 2 octas of cloud was reported for that day, and the wind speed decreased between $6 \mathrm{~h} 00$ and $8 \mathrm{~h} 00$.

Comparison of runners' UVR exposure and ambient UVR exposure. Runner's total UVR exposure was compared to the ambient solar UVR exposure for the identical periods of exposure. A spearman's correlation was run to determine the relationship between runners' UVR exposure and ambient solar UVR exposure. There was a strong, positive, statistically significant correlation between runners' UVR exposure and ambient UVR exposures $\left(\mathrm{r}_{\mathrm{s}}=\right.$ $0.7066, \mathrm{n}=8, \mathrm{p}=0.0501)$

Qualitative health risk results. Using the FST Classification, it is possible to determine a person's skin type and associated potential risk of sunburn. The first marathon runner who took part in the Cape Town half-marathon on 16 February could be described as having skin falling into the FST II category. This category includes skin types that are susceptible to sunburn with minimal solar UVR exposure. The first marathon runner received a total of 0.064 SED units/marathon. For sunburn to occur, the marathon runner required an exposure of 2.5-3 SED units of continuous exposure on unprotected skin. Therefore, in this instance, sunburn on unprotected skin was unlikely to have occurred on unprotected skin.

The second Cape Town half-marathon runner could be described as having skin classified according to the FST III category (i.e. white or light brown skin). This skin type may experience sunburn on regular sun exposure with no sun protection and the person is said to tan slowly. For this half-marathon, the runner received a total of 0.12 SED units/marathon. For sunburn to occur, the marathon runner required an exposure of 3-5 SED 
units of continuous exposure on unprotected skin. Therefore, in this instance, it is unlikely that sunburn occurred on unprotected skin.

The first runner who took part in the Pretoria half-marathon on 23 February could be described as having skin in the FST II category (i.e. white skin, very sensitive). This category includes skin types susceptible to sunburn with minimal solar UVR exposure. The marathon runner received a total solar UVR exposure of 0.11 SED units during the marathon. For sunburn to occur the marathon runner required an exposure of 2.5-3 SED units of continuous exposure on unprotected skin. Therefore, in this instance, sunburn was unlikely to have occurred on unprotected skin.

The second runner who took part in the Pretoria marathon could be described as having skin in the FST II category (white skin, very sensitive). The runner received a total solar UVR exposure of 0.055 SED units/marathon. For sunburn to occur the marathon runner required an exposure of 2.5-3 SED units of continuous exposure on unprotected skin. Therefore, in this instance, sunburn is unlikely to have occurred on unprotected skin. The runner that ran several training runs during a two-week training session in March/April and wore a UVR dosimeter badge had skin in the FST III category (i.e. white or light brown skin, moderately sensitive). The runner received an average of 0.03 SED units per training session (duration of training session varied). For sunburn to occur the runner required an exposure of 3-5 SED units of continuous exposure on unprotected skin. Therefore, in this instance, it is unlikely that sunburn occurred on unprotected skin during the training runs.

Hazard quotient results. The ambient total daily UVR exposure data for 2012 from the SAWS Pretoria site was used to calculate a marathon runner's typical solar UVR exposure over the course of a year. These data were combined with the calculated runner's solar UVR exposure as a percentage of the ambient UVR for marathons and for training 
sessions, respectively (as calculated from these study results), then superimposed onto a typical marathon runner's pattern of training session runs and marathon runs during the course of one, typical year. Table 5 presents the results of the HQ calculations. A full description of each variable and its calculation is provided in the data and methods section (see above).

Table 5. Calculation of the HQs for risk of acute health effects from solar UVR exposure during for marathon and training runs.

\begin{tabular}{|l|c|c|}
\hline Calculation & Marathon Runs & Training Runs \\
\hline Average (SED units) & 16.72 & 16.61 \\
\hline $\begin{array}{l}\text { Summed (cumulative) runner's solar UVR } \\
\text { exposure during one year (SED units) }\end{array}$ & 127.59 & 3.92 \\
\hline Number of days (n) & 24 & 152 \\
\hline Average exposure dose (SED units) each day & 5.31 & 0.25 \\
\hline Reference exposure level (SED units) & 1.08 & 1.08 \\
\hline Hazard quotient (HQ) & 4.922 & 0.024 \\
\hline Hazard risk according to Lemly & Moderate hazard risk & No hazard risk \\
\hline
\end{tabular}

The HQ calculations for the marathon and training runs differed. The number of marathons that were run throughout the year was 24 compared with 152 days of training. The training sessions took place mainly in the early mornings and late afternoons for approximately 105 minutes per session. Marathons had a different timing format compared to training sessions; however, the main difference between training and marathons was the distance that the marathons spanned. Marathons spanned distances of between $21 \mathrm{~km}$ to 89 $\mathrm{km}$ etc., which meant that the runner was exposed to the sun for extended periods of time during the course of the day depending on the marathon distance. Based on the guidelines provided by Lemly (19), the HQ for the runner's solar UVR exposure during marathon runs fell into the moderate risk category, while the HQ for the runner's solar UVR exposure during training runs fell into the no risk category. This is surprising yet expected, since although many more hours throughout the year were spent training compared with actual marathon running, the training runs typically took place during the early mornings and late afternoons when ambient solar UVR exposure levels are lower compared with those levels 
around midday, therefore the risk of sunburn, an acute effect, was lower during training compared to marathons.

\section{DISCUSSION}

South Africa is known to receive high levels of solar UVR. This was evident in the diurnal cycle of ambient solar UVR against which the SED values of the marathon runners' marathon and training runs were plotted. The 2012 yearly solar UVR cycle also depicted high amounts of solar UVR during the summer months (i.e., December, January and February) of South Africa. The runners' UVR exposure for the half-marathons that were monitored in Cape Town and Pretoria both showed low SED values. When these amounts were plotted against the diurnal cycle for the same day, it was evident that the values remained fairly constant and low, while the ambient solar UVR started to increase. The same trend could be seen for the runner's solar UVR exposure during the two-week training session. The low amounts of solar UVR received by the runners could be attributed to a number of factors, although in this study the meteorological factors did not seem to pose a significant emphasis on the low runners' SED values, however, with so few person-days this may not be calculable for all factors. One of the main factors that did contribute to the low SED values was the time of day at which the marathon runs and training runs took place. Both the marathon and training runs took place early in the mornings when the ambient solar UVR values were very low or close to zero. Hence, scheduling of marathons early in the morning (most probably for participant thermal comfort and safety rather than UVR considerations) may be an important planning method to reduce potentially high solar UVR exposure of runners. Another factor to be considered is the amount of shade along the marathon and training routes. Shade can decrease the amount of ambient solar UVR reaching an individual's body. While we did not record the amount of shade along the routes, this is an important parameter to record in future studies. 
When applying the FST Classification to assess sunburn risk for the runners, three of the four runners were FST II and the fourth runner was FST III. Both FST categories had some degree of sun sensitivity where the degree of the sensitivity is determined by the phenotypic characteristics of the runners and the amount of pre-determined SED values required to elicit a sunburn response. None of the four runners received SED values sufficiently high for sunburn to occur although prolonged exposure at these levels could lead to sunburn.

A typical year schedule for a marathon runner, including weekly training and actual marathon running, was drawn up for 2012 and the HQ was then calculated as an assessment of short-term acute health risks, separately, for the marathon and training runs to determine which may be more risky in terms of acute health risks. The solar UVR exposure patterns of the runners for marathons fell into the moderate hazard risk category, while those of the training runs fell into the no hazard risk category. Once again, the very small HQ calculated for the training runs can likely be attributed to the early morning and late afternoon training sessions, when the ambient solar UVR reaching the surface of the Earth is relatively low. However, the marathon runs resulted in a moderate hazard risk. This can be attributed to the marathons spanning much longer distances, hence prolonged periods of ambient solar UVR exposure for the runners. The personal solar UVR exposure of the four runners during marathon and training sessions did not yield high SED values and the associated risks were estimated as not being high or extreme. This was attributed to the timing of exposure, shade along the route and orientation of the dosimeter badge on the runner's body relative to the sun's direct rays. Given the prolonged periods of exposure runners may experience during full marathons that span a full day, potential health risks may be significant. Further research is needed to assess runners' solar UVR exposure during full marathons, as well as chronic 
solar UVR-related health risks, such as skin cancer, due to chronic sun exposure accumulated over a lifetime.

Feasibility and challenges. An important component of this study was to consider the feasibility of measuring marathon runners' personal solar UVR exposure during competitions and training sessions in South Africa. While we did execute the study with reasonable success, a number of challenges needed to be overcome. Recruitment of runners was difficult because some runners did not want to accept responsibility for looking after the UVR dosimeter badge. The runners were concerned that the badge would annoy and distract them during the run. This was the reason why we did not succeed in recruiting a runner to wear the UVR badge during the well-known Comrades Marathon run annually between Durban and Pietermaritzburg in May/June. The UVR badge could only be worn on the arm because of the Velcro strapping and configuration of the strap to the UVR badge. Given that the arms move continuously during running, and abrasion between the arm and torso may take place, this anatomic location of the UVR badge during running may not be ideal in terms of comfort and acceptability among runners. An alternate anatomic site to locate the UVR badge should be considered when measuring solar UVR exposure of runners.

Since a marathon route or training session route for runners may span several kilometres, finding a suitable site to measure ambient solar UVR and associated meteorological data is a challenge. This could be overcome, to some extent, by using the UVR badge for ambient monitoring along the marathon route. Cloud cover may vary significantly across a few kilometres and hence the distance between the runners' route and the ambient monitoring site may introduce bias to the results and subsequent calculations. Satellite data may be helpful to resolve some of these data constraints. In addition, premeasurement steps should be taken to deduce the best arm on which to attach the UVR badge 
(if this anatomic site remains the optimal choice) given the side of the road that the runner will run along and the direction of the sun rise, i.e. if the runner is running from south to north on the left-hand side of the road, the badge should probably be turned to face the east on the right arm so maximise personal solar UVR exposure measurements and capturing of data. Challenges also arise with the processing of ambient solar UVR data from multiple sites where logging intervals of the instruments differ and finding acceptable ways for data comparison is complex.

While we did apply the measured solar UVR exposure in the health risk calculations, since there was only a total 18 person-days of data, these results should not be considered representative of marathon runners' solar UVR exposure for marathons and training sessions and associated health risks in South Africa. A compounding problem was the relatively low personal solar UVR exposures of the runners for both marathons and training sessions. A more suitable method may apply for handling low solar UVR exposure data, and with more person-day data, one could apply the data to a chronic human health risk assessment model to estimate chronic health risks, such as skin cancer, based on a runner's cumulative lifetime solar UVR exposure patterns.

Acknowledgements - The authors would like to thank the Council for Scientific and Industrial Research, the Cancer Association of South Africa, the National Research Foundation for funding to support this research. New Zealand's National Institute for Water and Atmospheric Research are thanked for the loan of the personal solar UVR dosimeters. The South African Weather Services is thanked for the provision of data and for allowing the personal solar UVR dosimeters to be calibrated at their Head Office in Pretoria. The marathon runners who took part in the study are thanked for their contribution. 


\section{References}

1. Wright, CY., M. Norval, B. Summers, L. Davids, G. Coetzee and M. Oriowo (2012) Solar ultraviolet radiation exposure and human health in South Africa, Finding a balance. S. Afr. Med. J. 102, 665-666.

2. Armstrong, B. and A. Kricker (2001) The epidemiology of UV induced skin cancer. Photochem. Photobiol. B: Biol. 63, 8-18.

3. US Preventive Services Task Force (2012) Behavioural counselling to prevent skin cancer, recommendation statement. Available at: http://preventiveservicestaskforce.org/uspstf11/skincancouns/skincancounsrs.htm. Accessed on 8 January 2015.

4. Serrano, M.-A., J. Cañada and J.C. Moreno (2011) Ultraviolet exposure for different outdoor sports in Valencia, Spain. Photodermatol. Photoimmunol. Photomed. 27, 311-317.

5. Moehrle, M. (2001) Ultraviolet exposure in Ironman triathlon. Med. Sci. Sports. Exerc. 33, 1385-1386.

6. Ambros-Rudolph C.M., R. Hofmann-Wellenhof, E. Richtig, M. Muller-Furstner, H.P. Soyer and H. Kerl (2006) Malignant melanoma in marathon runners. Arch. Dermtol. 142, 14711474.

7. Moehrle, M. (2008) Outdoor sports and skin cancer. Clinics in Dermatol. 26, 12-15.

8. Allen, M and R. McKenzie (2013) Enhanced UV exposure on a ski-field compared with exposures at sea level. Photochem. Photobiol. Sci. 4, 429-437.

9. Wright, C.Y., A.I. Reeder, G.E. Bodeker, A. Gray and B. Cox (2007) Solar UVR exposure, concurrent activities and sun-protective practices among primary schoolchildren. Photochem. Photobiol. 83, 749-758.

10. McKenzie, R., B. Liley, P. Johnston, R. Scragg, A. Stewart, A.I. Reeder and M.W. Allen (2013) Small doses from artificial UV sources elucidate the photo-production of vitamin D. Photochem. Photobiol. Sci. 12, 1726-1737. 
11. Seckmeyer, G., M. Klingebiel, S. Riechelmann, I. Lohse, R. L. McKenzie, J. B. Liley, M. W. Allen, A.-M. Siani, and G. R. Casale (2012) A Critical assessment of two types of personal UV dosimeters. Photochem. Photobiol. 88(1), 215-222.

12. Seckmeyer, G., A. Bais, G. Bernhard, M. Blumthaler, C. R. Booth, K. Lantz, and R. L. McKenzie, Instruments to Measure Solar Ultraviolet Radiation. Part 2: Broadband Instruments Measuring Erythemally Weighted Solar Irradiance, World Meteorological Organization Global Atmosphere Watch, Report No. 164, WMO TD-No. 1289, Geneva, Switzerland, 55 pp., July 2008.

13. Swift, N., J. D. Hamlin, K. M. Nield, and R. L. McKenzie (2010) Characterisation of AlGaN detectors for UV measurements, paper presented at NIWA UV Workshop, Queenstown, 7-9 April. Available at: https://www.niwa.co.nz/sites/niwa.co.nz/files/algan detectors for uv.pdf

14. C.I.E. (Commission Internationale de l'Eclairage) (1997) A reference action spectrum for ultraviolet induced erythema in human skin. C.I.E.J. 6, 17-22.

15. C.I.E. (Commission Internationale de l'Eclairage) (1998) Erythemal reference action spectrum and standard erythema dose. C.I.E. S007/E-1998, CIE Central Bureau, Vienna, Austria.

16. Fitzpatrick, T.B. (1988) The validity and practicality of sun-reactive skin types I through IV. Arch. Dermatol. 124, 869-871.

17. N.R.C. (National Research Council) (1994) Science and judgment in risk assessment. Committee on risk assessment of hazardous air pollutants, Board of Environmental Studies and Toxicology, Commission on Life Sciences., pp 1-562, National Academy Press, Washington DC.

18. Moehrle, M., B. Dennenmoser and C. Garbie (2003) Continuous long-term monitoring of UV radiation in professional mountain guides reveals extremely high exposure. Int. J. Cancer. 103, $775-778$.

19. Lemly, A.D. (1996) Evaluation of the hazard quotient method for risk assessment of selenium. Ecotoxicol. and Environ. Safety. 35, 156-162. 


\section{Supplementary Data}

Table S1. A typical marathon runner's competition marathon schedule for Saturdays during 2012.

\begin{tabular}{|c|c|c|c|c|c|}
\hline Date & $\begin{array}{c}\text { Day of } \\
\text { the } \\
\text { Year }\end{array}$ & $\begin{array}{l}\text { Start } \\
\text { Time }\end{array}$ & End Time & $\begin{array}{c}\text { Duration of run } \\
\text { (hours) }\end{array}$ & $\begin{array}{l}\text { Distance of } \\
\text { marathon }\end{array}$ \\
\hline $2012 / 01 / 07$ & 7 & 06h00 & $08 \mathrm{~h} 00$ & 2 & $21 \mathrm{~km}$ \\
\hline $2012 / 01 / 21$ & 21 & 06h00 & $08 \mathrm{~h} 00$ & 2 & $21 \mathrm{~km}$ \\
\hline $2012 / 02 / 25$ & 56 & $06 \mathrm{~h} 00$ & $10 \mathrm{~h} 00$ & 4 & $42 \mathrm{~km}$ \\
\hline $2012 / 03 / 03$ & 63 & $06 \mathrm{~h} 00$ & 09h00 & 3 & $32 \mathrm{~km}$ \\
\hline $2012 / 03 / 10$ & 70 & 06h00 & $18 \mathrm{~h} 00$ & 12 & $72 \mathrm{~km}$ \\
\hline $2012 / 03 / 17$ & 77 & $06 \mathrm{~h} 00$ & $07 \mathrm{~h} 00$ & 1 & $21 \mathrm{~km}$ \\
\hline $2012 / 04 / 07$ & 98 & 06h00 & $12 \mathrm{~h} 00$ & 6 & $56 \mathrm{~km}$ \\
\hline $2012 / 04 / 14$ & 105 & $06 \mathrm{~h} 00$ & $11 \mathrm{~h} 00$ & 5 & $50 \mathrm{~km}$ \\
\hline $2012 / 05 / 05$ & 126 & $06 \mathrm{~h} 30$ & $10 \mathrm{~h} 30$ & 4 & $42 \mathrm{~km}$ \\
\hline $2012 / 06 / 02$ & 154 & $05 \mathrm{~h} 30$ & $15 \mathrm{~h} 30$ & 10 & $89 \mathrm{~km}$ \\
\hline $2012 / 07 / 07$ & 189 & $07 \mathrm{~h} 00$ & 09h00 & 2 & $21 \mathrm{~km}$ \\
\hline $2012 / 07 / 14$ & 196 & $07 \mathrm{~h} 00$ & $09 \mathrm{~h} 00$ & 2 & $21 \mathrm{~km}$ \\
\hline $2012 / 07 / 21$ & 203 & $07 \mathrm{~h} 00$ & $08 \mathrm{~h} 00$ & 1 & $10 \mathrm{~km}$ \\
\hline 2012/08/04 & 217 & $07 \mathrm{~h} 00$ & 09h00 & 2 & $21 \mathrm{~km}$ \\
\hline $2012 / 08 / 11$ & 224 & $07 \mathrm{~h} 00$ & 09h00 & 2 & $21 \mathrm{~km}$ \\
\hline $2012 / 08 / 18$ & 231 & $07 \mathrm{~h} 00$ & $08 \mathrm{~h} 00$ & 1 & $10 \mathrm{~km}$ \\
\hline $2012 / 09 / 15$ & 259 & 06h30 & $10 \mathrm{~h} 30$ & 4 & $42 \mathrm{~km}$ \\
\hline 2012/10/06 & 280 & $07 \mathrm{~h} 00$ & 09h00 & 2 & $21 \mathrm{~km}$ \\
\hline $2012 / 10 / 13$ & 287 & $07 \mathrm{~h} 00$ & 09h00 & 2 & $21 \mathrm{~km}$ \\
\hline $2012 / 10 / 20$ & 294 & $07 \mathrm{~h} 00$ & 08h00 & 1 & $10 \mathrm{~km}$ \\
\hline $2012 / 11 / 03$ & 308 & 06h00 & $12 \mathrm{~h} 00$ & 6 & $42 \mathrm{~km}$ \\
\hline $2012 / 12 / 08$ & 343 & $06 \mathrm{~h} 00$ & $08 \mathrm{~h} 00$ & 2 & $21 \mathrm{~km}$ \\
\hline $2012 / 12 / 15$ & 350 & $06 \mathrm{~h} 00$ & $08 \mathrm{~h} 00$ & 2 & $21 \mathrm{~km}$ \\
\hline
\end{tabular}

